Revue d'histoire de l'Amérique française

DE REVUE D.HISTOIRE DE L'AMÉRIQUE FRANÇAISE

\title{
Pierre, Jean-Baptiste, François-Xavier Le Gardeur de Repentigny (20 mai 1719 - 26 mai 1776) (suite et fin)
}

\author{
L. Jore
}

Volume 15, numéro 4, mars 1962

URI : https://id.erudit.org/iderudit/302155ar

DOI : https://doi.org/10.7202/302155ar

Aller au sommaire du numéro

Éditeur(s)

Institut d'histoire de l'Amérique française

ISSN

0035-2357 (imprimé)

1492-1383 (numérique)

Découvrir la revue

Citer cet article

Jore, L. (1962). Pierre, Jean-Baptiste, François-Xavier Le Gardeur de Repentigny (20 mai 1719 - 26 mai 1776) (suite et fin). Revue d'histoire de l'Amérique française, 15(4), 556-571. https://doi.org/10.7202/302155ar d'utilisation que vous pouvez consulter en ligne. 


\title{
PIERRE, JEAN-BAPTISTE, FRANÇOIS-XAVIER LE GARDEUR DE REPENTIGNY *
}

\author{
남
}

20 mai 1719 - 26 mai 1776

a.

Frère aîné de Louis Le Gardeur de Repentigny

Né à Montréal le 20 mai 1719,1 Pierre, Jean-Baptiste, François-Xavier de Repentigny entra très jeune dans l'armée; il fut promu successivement enseigne en second en 1734; en premier 1742 , lieutenant 1747. Sa carrière qui s'annonçait assez brillante faillit peu après être interrompue par un événement tragique dont il fut l'auteur responsable: le meurtre d'un commerçant de Québec nommé Nicolas, Jacques Philibert. Ce drame a fait l'objet d'un article publié par le juge canadien Baby, dans

* Voir notre Revue, XV : 64-89, 256-276, 396-418.

1 « Ce vingt quatrième may de mil sept cent dix neuf a êté batizé pierre Jean batiste francois Xavier né daujourdhuy fils de Mr. Jean batiste le gardeur darpentigny ecuyer officier aide major des troupes et de dame Marie Juchero son épouse, le parrain a été Mr Louis lienard de Beaujeu ecuyer capitaine du detachement de la marine et la marraine madame agathe St pair épouse de Mr. le gardeur darpentigny capitaine du detachement de la marine.

Signé: De Beaujeu, Agathe St pere, DE BELMONT prêtre.» Acte communiqué par M. l'Archiviste de la Cour Supérieure de Montréal. 
le Canadian Antiquarian and Numismatic Journal ${ }^{2}$ qu'a bien voulu nous signaler, avec son obligeance habituelle, le R.P. René Baudry, c.s.c.

«Notre intention, a écrit ce magistrat, n'est pas de faire ici l'histoire des Repentigny de la Nouvelle-Fance, mais tout bonnement de rectifier les faits d'un malheureux incident auquel se rattache le nom. Le romanesque, la poésie, se sont emparés de l'épisode et nous l'ont transmis plus ou moins altéré, varié, changé, selon le goût et la fantaisie de l'écrivain . . .

Dans l'intérêt de la vérité, de l'histoire, il est nécessaire de remettre autant que possible sous leur (son) véritable jour, cet épisode perdu dans les mystérieux nuages de la légende. ${ }^{3}$

Une lettre exhumée, il y a pas bien longtemps encore, nous met en mesure d'accomplir la chose, croyons-nous. Elle est de

2Sous le titre: «Mr. de Repentigny et le Chien d'Or», V: no 3 (Montreal, July 1898). Le R.P. René Baudry a bien voulu nous adresser sur le Chien d'Or, la note suivante:

« Cette histoire est bien connue à Québec, où une vieille maison existe encore, portant l'inscription suivante:

Je suis le chien qui ronge l'os;

En le rongeant je prends mon repos.

Un tems viendra qui n'est venu,

Où je mordrai qui m'a mordu !

L'interprétation de cette légende a donné naissance à plusieurs hypothèses. Placée sur une maison qui appartint à Philibert, elle aurait été apposée, selon les uns, par les enfants de Philibert comme un appel à la vengeance. On a dit aussi qu'elle aurait été apposée à la suite de démêlés entre Philibert et l'Intendant Bigot, et que même l'assassinat de Philibert aurait été provoqué par Bigot. Mais s'il est vrai que l'inscription date de 1736, comme l'avance Baby, elle n'eut rien à voir avec Bigot qui n'arriva à Québec que le 28 août 1748, année de la mort de Philibert. Il convient de remarquer que l'inscription en question se retrouve textuellement à Pézenas (Hérault) dès 1581, où elle était très connue. Philibert étant originaire de cette ville a bien pu la faire placer sur sa maison de Québec. 》

3 Il est assez singulier que des légendes prirent naissance, on ne sait comment ni pourquoi autour de ce drame. L'une d'elles prétendait qu'en tuant Philibert, Repentigny ne faisait qu'exécuter les ordres de l'Intendant Bigot. Or ce haut fonctionnaire n'arriva au Canada que huit mois après la mort de Philibert! Une autre veut que le meurtre eut pour origine l'amour que portait le fils du défunt à Amélie, fille de Repentigny et le refus de celui-ci de consentir au mariage. L'imagination de l'auteur de la légende l'avait emporté trop loin: en effet, Repentigny n'avait point de fille !

M. Roger Comeau, des Archives Canadiennes à Ottawa, nous signale aussi une étude de M. Pierre-Georges Roy, intitulée «L'Histoire vraie du Chien d'Or », publiée dans les Cahiers des Dix (Montréal, 1945). 
MM. Havy et Lefebvre, alors d'importants négociants à Québec, et adressée à Mr. Pierre Grey, le lendemain même, pour ainsi dire, de l'événement tragique...

Monsieur et cher ami.

Vous avez appris la fâcheuse nouvelle que le $\mathrm{Sr}$. Philibert a reçu un coup d'épée que Mr. de Repentigny lui a donné — dont il est mort trente six heures après. Leur difficulté est venue sur un billet de logement que Mr. le juge avait donné au dit Sr. de Repentigny pour le Sr. Philibert qui ne voulait pas loger chez lui et qui voulut payer la chambre où il logeait $6 \mathrm{f}$. par fois; l'hôtesse en demanda $10 \mathrm{f}$. Mr. de Repentigny qui était en haut, descendit. Il chargea Philibert d'injures comme faquin, sot, etc. Philibert répondit sans doute vivement à ces telles paroles. Enfin Mr. de Repentigny le poussa hors à la porte, et comme il s'en allait doucement et parlant fort en colère, $\mathrm{Mr}$. de Repentigny monte à sa chambre, va prendre son épée et court après Philibert et lui donne le malheureux coup dont il meurt Dimanche la nuit. Mr. de Repentigny a pris la fuite du costé de Montréal et le Sr. Panet ${ }^{4}$ est parti en poste Jeudy pour aller saisir tous ses biens. Celà fait une vilaine et malheureuse affaire. Il n'est pas douteux que le dit Sr. de Repentigny ne soit effigié en peu de temps. (Effigié, c'est-à-dire exécuté en effigie après un jugement par contumace).

Il paraît, continue le juge Baby, que la jeunesse dorée ${ }^{5}$ du temps ne se gênait guère de tirer l'épée pour bien peu, dironsnous, ces messieurs ajoutent:

Nous avons appris qu'à Montréal, les épées ont joué aussi, premièrement entre M.M. de Pensence ${ }^{6}$ et de

4 Huissier.

5 On attribue souvent à tort, et c'est le cas ici, ce nom à de jeunes oisifs fortunés. En réalité on l'avait donné en France, après le 9 Thermidor, aux membres (évidemment jeunes) d'un parti qui opposa des mours élégantes et des vêtements recherchés au laisser-aller de la faction démagogique, et se présenta comme hostile aux jacobins, vengeant les victimes de la Terreur. Cette réaction fut vaincue par Barras et Bonaparte (Dictionnaire historique des Institutions, mours et coutumes de la France). tonne.

6 Nous n'avons pu identifier cet officier certainement d'origine bre- 
Léry, ${ }^{7}$ et secondement entre M.M. de Jumonville ${ }^{8}$ et de la Bourdonnais. ${ }^{9}$ On dit qu'il y en a deux de très malades à l'hôpital. Ces messieurs se feront de mauvaises affaires ! Ils feraient mieux de réserver leur courage pour battre l'ennemi.

De fait, le Lieutenant d'Infanterie de Repentigny fut condamné par contumace par les Juges de la Prévôté de Québec à avoir la tête tranchée. En transmettant la procédure au Ministre, MM. de La Galissonnière ${ }^{10}$ et Bigot ${ }^{11}$ se bornèrent à écrire: "Vous verrés, Monseigneur, par les informations, de quoi il est question; il nous a paru par les circonstances du fait, que le cas lui-même est graciable, et nous ajouterons que cet officier s'étant distingué dans toutes les occasions de guerre depuis quatre ans et particulièrement à l'affaire des $《$ Mines $\gg^{12}$ et, en dernier lieu dans une expédition du côté d'Orange où il a défait un détachement considérable d'Anglais, dont vingt-six ont été tués et treize ont été emmenés prisonniers, il faut espérer les marques de clémence de Sa Majesté et nous vous suplions (sic) de les obtenir.

Le jugement par contrainte a été exécuté et la famille du Sr. Repentigni (sic) a satisfait à la partie civile, avec réserve néanmoins, au nom du Sr. Repentigni (sic) de se pourvoir par

7 Probablement Charles Chaussegros de Léry, né à Québec en 1728, décédé à Cayenne (Guyane) en 1767, frère de Mme Louis de Repentigny.

8 Un des frères Coulon de Jubinville. Nous n'avons pas pu savoir s'il s'agit en l'occurrence de celui qui fut tué en 1753 dans une embuscade par les soldats placés sous le commandement de Georges Washington, combattant alors comme officier anglais.

9 Parent, sans doute, de Mahé de La Bourdonnais qui se trouvait alors en Inde.

10 La Galissonnière (Roland, Michel, Marquis de) 1693-1756. Viceamiral, Gouverneur général du Canada.

11 Intendant du Canada. M. Guy Frégault lui a consacré un important travail intitulé François Bigot, administrateur français (2 vol. publiés par les Etudes de l'Institut d'Histoire de l'Amérique française, 261 Avenue Bloomfield, Outremont 8, Province de Québec, Canada).

12 La région dite des «Mines》 se trouvait dans le nord de la péninsule d'Acadie, aujourd'hui et depuis longtemps dénommée Nouvelle-Ecosse ou Nova Scotia, dont les Anglo-Américains avaient à plusieurs reprises tenté de prendre possession. 
la voye d'apel (sic) au Conseil Supérieur (lorsque le dit acusé (sic) se mettra en état) pour faire modérer les domages (sic) et intérêts, si elle ne peut les faire anéantir.»

On voit que la personne du pauvre Philibert ne comptait pas beaucoup aux yeux de MM. de La Galissonnière et Bigot ! Le Ministre pensait certainement de même puisqu'en mars 1749 il présentait au Roi une requête de clémence dans les termes suivants :

Le Sr. de Repentigny, Lieutenant dans les troupes du Canada demande des lettres de grâce pour l'homicide commis par lui sur la personne de Nicolas, Jacques, Philibert, négociant à Québec.

Le procès lui a été fait à la Prévôté de cette ville d'abord à la requête de Philibert lui-même, et la procédure ayant été continuée après son décès, à la requête de sa veuve, il a été condamné par un jugement du 20 mars 1748, à avoir la tête tranchée et 8000 Livres de dommages-intérêts envers la partie civile.

L'information est composée de six témoins. On y trouve que le Sr. de Repentigny ayant eu un billet pour aller loger chez Philibert, celui-ci s'en fut chez la nommée Lapalme, où cet officier logeait alors, et lui proposa de lui donner pour continuer de le loger 6 Livres par mois, mais que cette femme en voulait 10. Philibert dit que puisque celà était ainsi, il allait faire changer le billet parce que ses chambres étaient occupées par le service du Roy.

Que sur ces entrefaites, le Sr. de Repentigny ayant entendu Philibert qui parlait de faire changer son billet de logement, lui dit qu'il n'était qu'un nigaud et le mit à la porte en ajoutant: "Tu ne me connais pas, je t'aprendrais (sic) à me connaître.» Que Philibert luy a répondu « Je ne te connais pas $f$. gueux. 》

Qu'alors, le Sr. de Repentigny est rentré dans la chambre de la nommée Lapalme et est revenu sur le champ avec son épée, et, pendant que le 3ème témoin le retenait pour empêcher qu'il ne tombât sur Philibert, celui-ci a déchargé un coup de bâton qui 
aurait porté sur la tête de cet officier, s'il ne l'avait paré avec le bras. Que le Sr. de Repentigny s'est trouvé dans cet instant avoir la main droite libre et qu'il a allongé à Philibert un coup d'épée dans le côté gauche, dont il est mort.

Une autre main a ajouté sur la requête: «Mr. de La Galissonnière marque en particulier que le Sr. de Repentigny propose de partir en service aux isles, mais que cette proposition vient plutôt du chagrin que lui cause son affaire, que d'une envie sérieuse de changer de service, en sorte qu'à moins qu'on ne lui donne une Compagnie, je vous autorise à le laisser au Canada. » (Annotation non signée).

Pierre, Jean-Baptiste, François-Xavier de Repentigny obtint du Roy des Lettres de Grâce: «Celles-ci lui permirent, écrit le juge Baby, de reparaître dans les rues de Québec, mais il n'en demeura pas moins un sujet de mécontentement considérable pour une certaine classe très importante et très nombreuse de la Société qui ne pouvait lui pardonner aussi facilement que l'avait fait le Souverain, la mort de Philibert. Le Marquis de Jonquière, alors Gouverneur de la Nouvelle-France, en présence de l'opinion publique, crut devoir l'envoyer prendre du service en Acadie. » C'est à l'Ile Royale (aujourd'hui le Cap-Breton) qu'il fut affecté. Sa valeur militaire lui valut le grade de Capitaine en 1750 et la Croix de Chevalier de St-Louis en 1757. Comme son frère cadet Louis, il partit ensuite pour la France quand le Canada fut cédé à l'Angleterre et résida à Tours. Après un long temps de séjour dans cette ville, il finit par obtenir d'être présenté au Roi le 21 février 1769 par les Syndics et Directeurs de la Compagnie des Indes, pour continuer ses services dans les Etablissements des Indes Orientales ${ }^{13}$ avec le grade de LieutenantColonel et remplir les fonctions de Major Général, ainsi que celles de commandant en chef des troupes entretenues par la Compagnie qui comprenait un bataillon européen et un certain

13 Les possessions francaises dans l'Inde ne comprenaient plus depuis 1763 que les cinq villes de Pondichéry, Chandernagor, Karikal, Yanaon et Mahé, seules restituées par l'Angleterre qui s'était emparée de l'ensemble en 1761. 
nombre de formations de "cipayes » indigènes. ${ }^{14}$ Cette désignation ne fut pas du goût du gouverneur, François Law de Lauriston, ${ }^{15}$ qui exprima son opinion dans deux lettres adressées à M. Maineon d'Invault, ${ }^{16}$ contrôleur général des Finances et au Duc de Praslin. ${ }^{17}$

«Mr. de Repentigny notre Major Général, mandait celui-ci, dans le premier de ces documents, se donne certainement beaucoup de soin et de peine pour la discipline de la troupe, mais il ne peut donner aux officiers des connaissances qu'il n'a pas par raport (sic) au local. A peine dans ce corps d'officiers avonsnous un sujet qui connaisse le pays à une lieue hors de nos limites, pas un seul qui sache la langue ou qui ait la moindre teinture de la conduite qu'on doit tenir vis-à-vis des gens du pays. Si malheureusement la guerre survenait, dans quel embarras ne serions-nous pas ?»

La seconde lettre s'exprimait ainsi: «Je vous prie, Monseigneur, de faire attention à ce que j'ai eu l'honneur de vous marquer au sujet des officiers qui sont ici. Le tout est fort bon quand il ne s'agira que de deffendre (sic) cette place ou nos limites. Mais s'il s'agit par la suite d'envoyer dehors un fort

\footnotetext{
14 Voici copie du document consacrant cette décision: «Les Sindics (sic) et Directeurs de la Cie des Indes ayant jugé nécessaire de pourvoir à la place de Major Général et Commandant les Troupes entretenues par la dite Compagnie pour la garde de ses Etablissements aux Indes Orientales et Major de la Place à Pondichéry ont, en exécution de l'Edit de son Etablissement au mois d'Août 1764, confirmées par les Déclarations du Roy du mois de Février 1685 et 7 Septembre 1714 et notamment par l'Edit du mois de IMay 1719, portant réunion des Cies des Indes et de la Chine à celle d'Occident, à présent Cie des Indes, nomme et présente à $\mathrm{Sa}$ Majesté le $\mathrm{Sr}$ de Repentigny pour remplir la place de Major Général et Commandant les Troupes entretenues par la dite Cie pour la garde de ses Etablissemens aux Indes Orientales et Major de Place à Pondichéry sous les ordres du Commandant Général et du Conseil Supérieur à Pondichéry. Fait à Paris en l'Hôtel de la Cie des Indes le vingt et un Février mil sept cent soixante neuf. $\gg$ (Archives de France)

15 Parent du célèbre financier, F. Law de Lauriston (1724-1785) qui devint en 1780 Maréchal de Camp et Commandant en chef dans l'Inde.

16 Nous n'avons pas pu identifier ce personnage dont le nom a peutêtre été estropié dans la pièce d'archives où nous l'avons rencontré.

17 Le Duc de Praslin (1712-1785). Lieutenant général des Armées du Roi. Ambassadeur à Vienne. Duc et Pair en 1762. Occupa divers postes de Ministre mais fut entraîné dans la disgrâce de son frère le Duc de Choiseul.
} 
détachement ... il n'y a personne pour mettre à sa tête. Mr. de Repentigny quelque bon officier que je puisse le supposer ne connaît absolument rien au pays. De ce qu'il a couru au Canada de côté et d'autres, serait-il possible qu'on en eût conclu qu'il est propre à commander une armée dans l'Inde ? . . . Il peut le devenir, sans doute, mais pour celà, il faudra qu'il soit d'abord en sous-ordre ou suposé (sic) qu'il commande, qu'il se laisse entièrement guider par des officiers qu'il aurait sous lui . . . Le seul parmi les officiers qui sont ici qu'on puisse dire instruit et qui serait en état de commander un corps de troupes en campagne est Mr. Beylié, capitaine, commandant l'artillerie de cette place. »

Le Ministre ne tint aucun compte de ces remarques et Repentigny continua à occuper le poste qui lui avait été confié. A vrai dire, il était probablement impossible de le remplacer par un autre officier remplissant les qualités que Law de Lauriston estimait que le Major Général devait posséder et il ne pouvait sans doute pas être question de promouvoir le Capitaine Beylié à un grade supérieur. Une autre difficulté surgissait du fait qu'un officier d'artillerie nommé Lagrenée avait cru devoir, dans une lettre adressée le 10 octobre 1771 aux Syndics de la Compagnie des Indes, faire remarquer que c'est lui et non Repentigny qui devrait remplacer le Gouverneur (on ne sait sur quels titres) si celui-ci venait à décéder. Il ne semble pas que cette lettre ait été suivie d'une réponse; aussi bien la question du remplacement de Lauriston qui ne songeait point à trépasser, ne se posait-elle pas. Lagrenée ne se doutait pas que le 28 mars 1771, les Directeurs de la Cie des Indes étaient intervenus auprès du Ministre pour qu'il voulut bien faire promouvoir Repentigny au grade de Colonel.

Permettez-nous, Monseigneur, de vous exposer . . . que nous croyons Mr. de Repentigny, LieutenantColonel, Major Général des Troupes de l'Inde, dans le cas d'espérer un brevet de Colonel. Cet Officier a très bien servi en France et au Canada. C'est d'après les meilleurs témoignages qu'il a été choisi pour commander les troupes qui sont entretenues dans l'Inde. Nous le croyons digne de la grâce que 
nous demandons pour lui. Un grade supérieur ne peut que lui procurer une plus grande considération; la distance qu'il mettra entre lui et les officiers qui sont sous ses ordres assurera encore plus la diférence et la subordination si nécessaires au bien du service. Nous croyons aussi, Monseigneur, devoir vous faire observer que c'est un moyen de retenir cet officier dans l'Inde et qu'il est intéressant de l'y fixer pour profiter de ses talents et de son zèle.

Le 17 mars 1772, le Ministre informait le Gouverneur des Établissements Français dans l'Inde de l'élévation au grade de Colonel dès le mois de mai de l'année précédente (pourquoi avaiton tant attendu ?) du Lt-Colonel de Repentigny. « Je joins icy, ajoutait-il, la commission qui lui en a été expédiée. Vous aurez à la lui remettre en cette qualité. »

Une seconde dépêche de même date, adressée à Repentigny lui-même était ainsi conçue :

« Je vous préviens, Monsieur, que j'adresse à Mr. Law la commission de Colonel que le Roy a bien voulu vous accorder. Je ne doute pas que cette grâce ne vous engage à continuer de donner des preuves de votre zèle pour le servir.»

L'année suivante, lorsque le Bataillon de l'Inde fut reformé et que fut créé à sa place un Régiment, le Ministre, en portant cette décision le 20 février 1773 à la connaissance du Gouverneur, lui fit savoir que cette nouvelle unité porterait le nom de Régiment de Pondichéry ${ }^{18}$ et que le Roi en avait nommé Repentigny colonel. «Toutefois, précisait-il, dans la notification faite à l'intéressé, cette commission ne doit avoir aucun effet et Sa Majesté ne vous l'a accordée que pour confirmer encore plus expressément un grade qu'elle a déjà eu la bonté de vous conférer.

18 Le nouveau régiment de Pondichéry fut créé par une Ordonnance du 30 déc. 1772. Il devait comprendre 2 bataillons de 9 compagnies chacun, dont une de grenadiers et huit de fusiliers. L'Etat-Major aurait 1 Colonel, 1 Lt-Colonel, 1 Major, 1 Aide-Major et un Sous-aide major par bataillon, un quartier-maitre et deux porte-drapeaux par bataillon. Les parements, les collets et les revers devaient être de drap couleur orangé, les boutons timbrés d'une ancre. Les grenadiers se distinguaient par deux épaulettes de drap de même couleur que le parement ( $D$ 'après Louis Baudzat, La formation de l'armée coloniale). 
Sa Majesté vous donne donc dans ce moment-cy une marque beaucoup plus sensible de la confiance et de la satisfaction qu'elle a de vos services, en vous nommant à Chandernagor pendant l'absence de Mr. Chevalier qui a demandé un congé pour rentrer en France.» Mais deux mois plus tard (17 avril 1773), le Ministre annonçait à Repentigny que la mutation annoncée ne serait suivie d'aucun effet, M. Chevalier ayant renoncé à son congé. En échange, poursuivait-il, « vous pourrez employer utilement le séjour que vous ferez dans l'Inde en visitant les Établissements Français en sus de Pondichéry pour en faire connaître la véritable situation et le parti qu'on peut en tirer. J'écris à Mr. Law de vous procurer les moyens que vous lui demanderés pour rendre l'inspection que vous ferez la plus utile qu'il soit possible. Lorsqu'elle sera finie, il donnera les ordres nécessaires pour votre retour en France et, en attendant, vous continuerez de jouir du traitement que vous aurez comme Major Général Commandant le Bataillon de l'Inde. »

Les deux correspondances précitées marquent clairement le désir que l'on avait à Paris, vraisemblablement à la demande du Gouverneur, d'éloigner Repentigny de Pondichéry, mais aussi l'embarras qu'on éprouvait de prendre une pareille mesure, l'intéressé n'ayant pas démérité. On ne trouve rien dans les archives qui puisse expliquer les motifs de celle-ci. Il est à penser, d'autre part, que Repentigny ne se souciait nullement d'exécuter la tournée d'inspection dont on le chargeait et que c'est avec une grande satisfaction qu'il accepta d'aller remplacer M. DuPrat, Commandant particulier à Mahé et Commandant de l'Établissement français à la Côte de Malabar lequel, selon Lauriston, y avait commis des imprudences. Il ne devait séjourner que peu de temps à Mahé. Le 16 février 1775, le Ministre de la Marine l'invitait à remettre le service à M. Picot, (prédécesseur de M. DuPrat) qui avait été reconnu comme ayant bien agi et qui en conséquence avait été relevé à tort de ses fonctions. En lui confirmant cette décision par dépêche du 5 mars 1775, le Ministre ajoutait:

"Il me reste à vous témoigner la satisfaction de Sa Majesté pour le zèle avec lequel vous vous êtes porté à exécuter ce 
commandement après le rapel (sic) de Mr. le Comte du Prat et sur la manière dont vous en avés rempli les fonctions. Je me suis fais rendre compte à cette occasion de ce qui vous concerne et je me propose d'en mettre incessamment le détail sous les yeux du Roy. L'intention de Sa Majesté est maintenant que lorsque vous aurés remis le commandement de Mahé à Mr. Picot, vous vous rendiez à Pondichéry auprès de Mr. Law à qui je ferai passer ultérieurement les ordres de Sa Majesté en ce qui vous regarde. $\gg^{19}$

Le 6 décembre 1775 Repentigny rendait compte au Gouverneur de l'arrivée de $\mathrm{M}$. Picot à Mahé le 12 novembre précédent et qu'il l'avait fait reconnaître en qualité de Commandant pour le Roi de ce comptoir. Nous n'avons pu déterminer la date à laquelle Repentigny arriva à Pondichéry. Sa santé devait être assez sérieusement atteinte depuis quelque temps déjà puisque dès le mois de mai 1775 , Lauriston l'avait autorisé à remettre le service à son second et à revenir au chef-lieu, permission dont il n'avait pas cru devoir user. Sans doute ne reprit-il aucune fonction. L'acte de son décès survenu à Pondichéry le 26 mai 1776 se borne en effet à enregistrer ses titres. ${ }^{20}$

19 On est mal renseigné sur les activités de Pierre de Repentigny à Mahé. Une lettre de Law de Lauriston au Ministre, datée du 1er juin 1775, nous apprend seulement qu'il y fit une expédition: «Après la lettre de $\mathrm{Mr}$ de Repentigny et la mienne du 28 janvier où je lui recommandais de ne rien compromettre, de bien peser ses forces avant d'agir, cette lettre me mit dans la plus grande inquiétude. Au reste, ce n'est pas que je prétende désapprouver la conduite de $\mathrm{Mr}$. de Repentigny. Il aura sans doute, comme je le lui avais prescrit pezé (sic) et examiné ses forces ainsi que celles de l'ennemi, mais n'ayant jamais fait la guerre dans l'Inde, Mr. de Repentigny était, et je crois ne l'est plus, comme beaucoup d'officiers qui arrivent d'Europe ayant le plus grand mépris pour tout ce qui s'appelle noir et s'imaginant qu'il suffit de se présenter pour les faire fuir. Des gens qui se font tuer et qui bravent la mort ne sont jamais à mépriser quelque ignorants qu'ils soient dans l'art de la guerre, mais d'ailleurs, ce qui justifie entièrement Mr. de Repentigny, c'est qu'il paraît par ses lettres que Chériquel ne voulant entendre à aucune proposition, l'a mis dans le cas forcé d'agir. ... Les lettres de $\mathrm{Mr}$. de Repentigny des 4 et 28 avril font voir comment cette petite guerre a été terminée... Mr. de Repentigny en a profit, une trève ayant été établie le 2 avril. 》

20 Acte de décès du Colonel Pierre Le Gardeur de Repentigny : «Aujourd'hui vingt sept may mil sept cent soixante seize, je soussigné certifie avoir enterré dans le cimetière de l'église paroissiale notre dame des Anges de Pondichery messire Pierre LEGARDEUR de REPENTIGNY colonel d'infanterie chevalier de l'ordre royal et militaire de Saint Louis cy devant 
Ainsi disparut loin des siens, à l'âge de 57 ans, après un séjour ininterrompu de six années aux Indes, ce loyal serviteur du Roi et de la France. Avait-il regretté plus que personne autant que veut bien le dire le juge Baby, l'acte inqualifiable que la colère et l'emportement l'avaient poussé inconsidérément à commettre dans sa jeunesse ? On peut le supposer, puisque, selon le même auteur, tous s'accordaient à lui reconnaître un caractère généreux et doué d'une grande bonté d'âme.

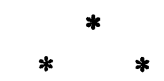

Pierre, Jean-Baptiste, François-Xavier de Repentigny avait épousé à Montréal, le 30 janvier 1753, Catherine, Angélique, fille de Pierre, Jacques Payant de Nohan et de Louise d'Ailleboust de Manthet, qui mourut à Lachenaie le 17 décembre 1757. De cette union étaient nés deux enfants décédés l'un et l'autre le jour même de leur venue au monde, respectivement le 28 décembre 1755 et 9 novembre 1757. Le 26 juin 1766, en l'église St-Vincent de Tours, ville dans laquelle il séjournait avec nombre d'autres officiers canadiens et en particulier près de son frère cadet Louis ainsi que de la femme de ce dernier, FrançoisXavier de Repentigny avait épousé en secondes noces Marguerite, Jeanne Mignon, fille de Philippe, Jean-Baptiste Mignon, Procureur du Roi au Bureau des Finances de la Généralité de Tours ${ }^{21}$ qui lui donna deux enfants, un fils: François-Xavier et une fille : Marguerite, Madeleine.

Né à Tours le 17 novembre 1767 le premier n'avait que neuf ans quand son père mourut à Pondichéry. Sa mère se mit en

major général des troupes de l'Inde, ancien commandant pour le Roy à Mahé, né au Canada. Il est mort le vingt six du même mois et an que dessus après avoir reçu les sacrements, agé d'environ cinquante cinq ans, ont signé comme témoins messire Jean Baptiste de LA SALLE MARIEHAURE, capitaine d'infanterie, natif de Paris, paroisse Saint André des Arts, François NICOLAS, natif de Pondichery qui ont signé avec moi. Signé: DELASALLE MARIEHAURE, F. NICOLAS, et frère Sebastien de Nevers, capuçin missionnaire apostolique, curé. $\gg$ (Dépôt des Papiers Publics des Départements et Territoires d'Outre-Mer. Etablissements français dans l'Inde - Pondichéry - Année 1776 - $27 \mathrm{mai}$ ).

21 Il est assez singulier que dans son acte de mariage, il soit qualifié du titre de Lieutenant des Vaisseaux du Roy. 
instance en 1777 auprès des autorités pour le faire admettre dans une Ecole Militaire. ${ }^{22}$ Le Département des Manuscrits à la Bibliothèque Nationale conserve la trace de cette requête dont l'examen rencontra des difficultés quant à la preuve de noblesse du candidat. ${ }^{23}$ Soumise à l'appréciation du Prince de Montbarey, Ministre de la Guerre, la question posée donna lieu à la réponse suivante:

J'ai rendu compte au Roy, Monsieur, de l'embarras où se trouve Mme de Repentigny pour produire les titres et la filiation de trois générations faisant preuve de noblesse que vous lui demandés pour pouvoir lui délivrer le certificat qui lui est nécessaire pour conduire à sa destination son fils qui a été nommé Elève au dernier travail (d'admission aux Ecoles Militaires) et Sa Majesté, considérant que le recouvrement de ces titres qui sont restés à Québec où ses ayeux sont morts au service, serait très long et très difficile, a bien voulu approuver, sans tirer à conséquence, que vous délivriez le certificat de noblesse qu'elle sollicite, sur les titres primordiaux qu'elle nous a adressés. Vous aurez agréable de vous conformer à cet égard aux intentions de Sa Majesté et de m'envoyer ce certificat le plus promptement qu'il vous sera possible.

Ce certificat fut ainsi conçu :

Produisant : Pierre François Xavier Le Gardeur de Repentigny, né le 19 Novembre (17?) 1767, en légitime mariage du dit François (Xavier) Le Gardeur de Repentigny et de la dite Dame Marguerite, Jeanne Mignon, son épouse, et batisé (sic) le même jour en la paroisse de St. Vincent de la Ville de Tours, a été agréé par le Roi pour les Ecoles Militaires le 31 Décembre 1777. En foi de quoi, etc.

22 En dépit de leur dénomination, les Ecoles Royales Militaires étaient tout simplement des Etablissements d'enseignement secondaire dirigés par des religieux de divers ordres secondés occasionnellement par des professeurs laîques. Elles étaient au nombre de dix. Leur nom venait du fait que les jeunes gens appartenant à la noblesse qui y étaient admis entraient dans l'armée à la fin de leurs études. Les élèves portaient un uniforme.

23 Lettre de d'Hozier de Sérigny au Prince de Montbarey, Ministre de la Guerre, du 25 janvier 1778. 
Nous n'avons pas pu savoir si l'enfant fut admis dans une des Ecoles Militaires ni dans laquelle. En 1782, à l'âge de quinze ans, il était nommé aspirant, puis, le 31 juillet 1784, "gardemarine" ${ }^{24}$ au Département de Rochefort et cinq mois plus tard embarqué sur le David à bord duquel il accomplit une campagne aux Iles-Sous-le-Vent (Antilles) jusqu'en mai 1786; passé sur la Seine à cette époque, il fit partie de l'escadre d'évolution du Vicomte de Beaumont. En 1787-1788, il participa à une croisière sur la Fauvette aux Côtes d'Afrique et sur la Bienvenue et la Thétis à une campagne aux Indes Orientales en 1791.25 Revenu en France en décembre de cette dernière année, il émigra et s'enrôla dans l'Armée des Princes où il servit dans la cavalerie. Officier au Régiment d'Hector, il prit part avec ce corps à la funeste expédition de Quiberon. "Echappé au désastre, incorporé avec les débris d'Hector dans Mortenart, gratifié du rang de Major d'Infanterie, il ne tarda pas à se lasser de ce service décevant. En 1796, il coopérait avec les Uhlans Britanniques à la prise de Sainte-Lucie. Ce fut cette année là qu'il obtint de rester à la Martinique, alors anglaise. Après une nouvelle campagne à Sainte-Lucie, une des plus pénibles et meurtrières, il revint à la Martinique. De 1797 à 1802, il s'employa activement dans les dragons-milices du Lamentin jusqu'au moment où le rétablissement de la paix lui permit de rentrer en France. ${ }^{26}$ Tout naturellement, il se fixa à Tours, lieu de naissance de sa mère et le sien et où il épousa Marie Jacques (sic) Delphine Gaigneron Jollinon Desmarais. Point n'est besoin de dire qu'il accueillit avec enthousiasme le retour des Bourbons. Dès le 5 juillet 1814, il recevait la Croix de St-Louis et le 31 décembre

24 Colbert avait créé en 1670 à Brest, Rochefort et Toulon, trois compagnies de Gardes de la Marine dans lesquelles seraient recrutés les officiers de Marine. Ces jeunes gens servaient comme simples soldats et faisaient en même temps des études pour se préparer à cette carrière. Ils portaient un habit de drap bleu dont les parements étaient rouges comme la veste (gilet) la culotte et les bas. M. Marion, Dictionnaire des Institutions de la France aux XVII et XVIIIIes siècles (Paris, Picard 1923). 25 C. Bonnault de Mézy, «Les Canadiens en France et aux Colonies après la cession, 》 Revue de l'Histoire des Colonies françaises, 1924.

26 Ibid. 
suivant le brevet de Capitaine de "Vaisseau ${ }^{27}$ et une pension de retraite. Plus tard il fut nommé maire de Genillé, commune de 2,000 habitants située dans l'arrondissement de Loches, où il possédait vraisemblablement une propriété, et fit partie du Conseil Général du Département d'Indre-et-Loire.

Pierre, François-Xavier de Repentigny mourut à Tours le 4 décembre 1820, âgé de 52 ans seulement. Le 7 du même mois le Journal d'Indre-et-Loire fit part à ses lecteurs de son décès et consacra au défunt une élogieuse notice. Il laissait une fille, Xavérine, née à Tours le 28 Vendémiaire an XIII (28 octobre 1804) qui épousa le Comte Charles Urbain Hélion de Barbançois.

La fille du Colonel Pierre, Jean-Baptiste, François-Xavier de Repentigny et de Marguerite Jeanne Mignon, Marguerite Madeleine, née à Tours le 27 mars 1769, avait été confiée par son père lorsqu'il partit pour les Indes cette même année, aux Dames Ursulines à Tours avec ordre au Notaire Beauregard de se charger de ses affaires et de pourvoir à ses besoins. Etant donné que la fillette venait de naître, on est en droit d'être surpris d'une telle mesure puisque sa mère était vivante. Il est non moins curieux de constater que le 2 mai 1775 ce fut son oncle Louis de Repentigny, alors Colonel en service à Rochefort, qui écrivit au Ministre de la Guerre pour solliciter en sa faveur une pension annuelle de 1,250 francs, à valoir sur les appointements de son père, absent pour le service Outre-mer, le Notaire Beauregard ayant fait banqueroute; ce à quoi le Ministre consentit sans faire de difficultés.

Le 12 mai 1777, se trouvant à Basse-Terre, et apprenant le décès de son frère à Pondichéry un an plus tôt, ce fut encore Louis de Repentigny qui intervint de nouveau auprès du Ministre

${ }^{27}$ Il n'avait jamais reçu le grade de Capitaine de Frégate avant d'émigrer, comme le prétend le rédacteur de sa nécrologie parue dans le Journal d'Indre-et-Loire, n'ayant servi dans la marine que trop peu de temps. Il est possible que l'auteur de la notice ait confondu les grades des officiers de marine. 
pour que l'on attribuât à Marguerite Madeleine une pension. Le 17 février 1789, la jeune fille épousa à Tours, en l'église St-Vincent, Louis Augustin de Villeneuve de Cazeau d'une ancienne famille de la région.

(Fin)

L. JORE,

Gouverneur général $h^{T e}$ de la France d'Outre-mer, Ancien gouverneur du Sénégal et dépendances 